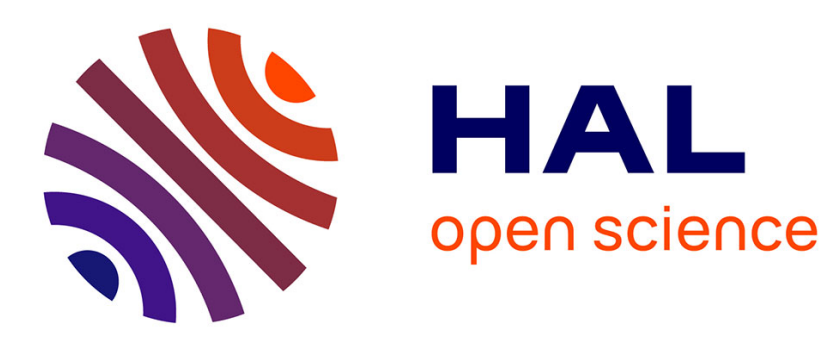

\title{
Organiser les situations de travail pour l'apprentissage: approche didactique de la formation professionnelle par alternance.
}

Laurent Veillard

\section{- To cite this version:}

Laurent Veillard. Organiser les situations de travail pour l'apprentissage: approche didactique de la formation professionnelle par alternance.. Durand, M.; Fillietaz, L. Travail et formation des adultes, PUF, pp.125-156, 2009, 9782130570431. halshs-00376335

\section{HAL Id: halshs-00376335 \\ https://shs.hal.science/halshs-00376335}

Submitted on 21 Oct 2009

HAL is a multi-disciplinary open access archive for the deposit and dissemination of scientific research documents, whether they are published or not. The documents may come from teaching and research institutions in France or abroad, or from public or private research centers.
L'archive ouverte pluridisciplinaire HAL, est destinée au dépôt et à la diffusion de documents scientifiques de niveau recherche, publiés ou non, émanant des établissements d'enseignement et de recherche français ou étrangers, des laboratoires publics ou privés. 


\title{
Organiser les situations de travail pour l'apprentissage : approche didactique de la formation professionnelle par alternance.
}

\author{
Laurent Veillard - laurent.veillard@univ-lyon2.fr \\ UMR ICAR (CNRS - Université de Lyon)
}

En France, le développement récent de l'alternance à tous les niveaux de formation a favorisé l'émergence d'un nouveau champ de réflexion et de recherche en éducation (Chaix, 1993 ; Cohen-Scali, 2000 ; Geay, 2000 ; Besson, Colin, Geay \& Hahn, 2005). Une des problématiques souvent discutée est relative au partenariat entre école et entreprise au niveau de l'organisation pédagogique de la formation. Plusieurs classifications ont été proposées pour tenter de caractériser différentes formes d'alternance en fonction du degré de collaboration pédagogique entre l'école et l'entreprise (Lerbet, 1993; Maubant, 1994). Ces travaux concluent à une collaboration minimale dans de nombreux cas, qui se réduit à une juxtaposition entre une formation théorique et technique à l'école et des apprentissages pratiques sur le lieu de travail.

Certains préconisent d'aller au delà pour développer un pédagogie propre à l'alternance. Ils proposent de prendre acte que cette modalité pédagogique rassemble deux sphères très différentes, l'une où le savoir a une valeur en lui-même alors qu'en entreprise il ne vaut que par ce qu'il permet de faire. Ils considèrent aussi, dans une perspective piagétienne, que la pratique ou l'expérience en situation de travail comporte son propre niveau d'autonomie en tant que source de savoirs spécifiques indispensables à la compétence (Besson, Colin, Geay \& Hahn, 2005). Dans le même ordre d'idée, des travaux anglo-saxons montrent que de nombreuses institutions professionnelles, tant artisanales qu'industrielles, ont une logique propre d'intégration et de formation des novices, basée sur des principes tels que : l'allocation de séquences de tâches de complexité et de responsabilités croissantes; la participation progressive à des collectifs de travail ; la mise en place de processus de guidage des plus expérimentés vers les nouveaux venus (Lave \& Wenger, 1991 ; Darrah, 1996). On apprend donc autant à l'école que sur un lieu de travail mais selon des logiques différentes et autonomes. Partant de là, une pédagogie de l'alternance revient essentiellement à construire des médiations entre ces deux types de situations d'apprentissage, pour aider les apprenants à transformer les savoirs d'expérience acquis sur le terrain professionnel en savoirs transférables et lier les différents types de savoirs (théoriques, techniques, disciplinaires et d'expérience) entre eux. (Besson, Colin, Geay \& Hahn, 2005)

Sans remettre en cause l'importance de ces médiations, on peut aussi partir de l'hypothèse que les institutions professionnelles ont parfois des difficultés pour accueillir et former des apprenants, par exemple parce qu'elles le font pour la première fois, ou parce qu'elles ont peu de temps et de moyens pour cela. Se posent également les questions de la cohérence des apprentissages au travail et à l'école et celles de l'exhaustivité et de la validité et des acquisitions sur le terrain professionnel. Car des expériences sur des lieux de travail peuvent tout à fait conduire à des apprentissages partiels, voire incorrects, notamment lorsqu'ils sont basés sur la reproduction implicite de routines d'acteurs expérimentés sans compréhension des raccourcis opérés, ou encore de tours de main enfreignant les règlements (Billett, 2000). Une réponse possible à ces problèmes est de sortir du principe de relative autonomie des deux institutions pour considérer qu'un dialogue est nécessaire entre elles sur le plan pédagogique. 
La collaboration peut se faire par le biais d'un pilotage par l'amont, comme dans le système dual allemand et suisse où les responsabilités des deux partenaires de la formation sont clairement séparées et fixées dans des cahiers des charges précis : l'école a la responsabilité de la formation théorique et technique générale; les entreprises ont un cahier des charges très précis des compétences à développer et des situations d'apprentissage à mettre en œuvre. En France, dans les dispositifs par apprentissage, seule la loi générale fait référence et impose peu de contraintes : donner des tâches en rapport avec l'objectif de la formation; créer des conditions favorables pour que le jeune apprenti puisse apprendre ; désigner un tuteur entreprise (articles L117-7 et L117-4 du code du travail). La cohérence et la complémentarité des deux espaces-temps de la formation doit être négociée entre l'école et l'entreprise à chaque nouveau contrat d'apprentissage, en particulier par un dialogue entre le tuteur école et le tuteur entreprise de chaque apprenti Cependant, de telles négociations dépendent beaucoup du savoir-faire des deux tuteurs. Pour tenter d'aider les tuteurs et d'harmoniser autant que possible les situations en entreprise, plusieurs formations tentent de mettre en place un cadre d'organisation générale de l'alternance.

Dans, ce chapitre, nous nous intéressons à la manière dont une institution de formation en alternance d'ingénieurs tente de cadrer les situations et les tâches confiées aux apprentis dans les entreprises. Quelle organisation a-t-elle mise en place pour cela ? Quels sont les effets de cette organisation sur les processus effectifs de transmission de savoirs sur le terrain professionnel et finalement les apprentissages des apprentis ? Nous proposons d'abord un cadre théorique issu de la didactique disciplinaire, qui nous semble apte à traiter de ces questions. Puis nous nous appuyons sur ce cadre pour analyser deux études de cas réalisées dans l'école d'ingénieur par apprentissage.

\section{Approche théorique}

Notre cadre théorique est principalement basé sur le travail de Chevallard (1992, 2003), qui luimême s'appuie sur les œuvres de Durkheim (1893) et Douglas (1987). Chevallard fonde sa théorie anthropologique du didactique sur quelques notions fondamentales : objet; personne; rapport à un objet; institution. "Est objet [de connaissance] toute entité, matérielle ou immatérielle, qui existe pour au moins un individu. Tout est donc objet, y compris les personnes ». (Chevallard, 2003, p. 81). Une personne, dans sa vie personnelle et professionnelle, va rencontrer des objets, créer et modifier des rapports pratiques, cognitifs, émotionnels avec ceux-ci. Apprendre est défini ici comme la création ou la modification de rapports à des artefacts culturels ou physiques, des personnes, par interaction avec eux. Une institution est un dispositif social total parfois de durée de vie et d'extension sociale réduite, qui permet et prescrit aux personnes qui viennent y occuper une certaine position, la mise en jeu de manières de faire et de penser propres (ibidem, p. 82). Ainsi, une école d'ingénieur, une classe, une entreprise ou un service au sein de celle-ci, est une institution. En clair, une institution définit un certain nombre de positions en son sein, et demande aux personnes qui en deviennent membres (ses sujets) de constituer des rapports spécifiques à des objets, rapports qui dépendent pour une large part de ces positions mais pas seulement. Les sujets d'une institution gardent toujours une marge de manœuvre et des façons propres d'y occuper des positions, en particulier parce que leurs " assujettissements allogènes (engendrés par d'autres institutions) travaillent leurs rapports personnels, souterrainement peut-on dire (du point de vue de l'institution), en y inscrivant leur coloration propre " (Chevallard, 1992, p. 92) 
Toute institution organise une part plus ou moins grande d'instruction car les connaissances nécessaires pour accéder aux différentes positions en son sein ne peuvent généralement pas procéder uniquement d'une volonté personnelle et d'un apprentissage par l'action individuelle (Billet, 2000). La théorie anthropologique du didactique propose de modéliser ces processus d'instruction institutionnelle à l'aide de la notion de système didactique. Un système didactique se constitue, au sein d'une institution, pour une durée plus ou moins longue, lorsque : un ou des sujets de l'institution viennent occuper une position d'enseignant; un ou des sujets viennent y occuper une position d'apprenant; un enjeu didactique, (créer ou modifier le rapport à un ou des objets) est défini pour le pôle apprenant. Les systèmes didactiques sont évidemment omniprésents dans les institutions dédiées à l'enseignement ou à la formation, mais ils peuvent aussi se former fréquemment dans des institutions de production de biens ou de services, de manière formelle ou informelle, par exemple à l'occasion d'un processus d'intégration de nouveaux venus (tutelle d'un plus ancien) ou lors d'une formation du personnel.

Chevallard avance deux conditions nécessaires pour qu'un système didactique fonctionne, c'està-dire joue son rôle de transmission et permette des apprentissages. Il faut d'abord que se mette en place un contrat didactique entre les acteurs du système, c'est-à-dire un ensemble d'attentes et d'obligations réciproques, qui peuvent être largement implicites, sur ce que chacun doit faire. Il faut également qu'un ensemble d'objets aillent de soi et soient localement stables pour les acteurs du système et constituent ainsi un milieu d'objets partagé pour que l'activité d'enseignementapprentissage puisse avoir lieu.

Dans notre étude, nous nous intéressons plus particulièrement aux systèmes didactiques qui se mettent en place au sein de l'institution entreprise autour d'un apprenti en formation en alternance. Nous étudions en premier lieu les moyens pris par l'institution école pour intervenir sur ces systèmes didactiques qui se déroulent dans une autre institution. Nous partons de l'idée que ces moyens sont principalement des prescriptions ou recommandations adressées aux entreprises, mais que celles-ci peuvent les adapter, voire refuser de les appliquer en fonction de leurs contraintes propres. En psychologie du travail, l'analyse des rapports entre le prescrit et le réel passe en particulier par l'étude des liens entre tâche prescrite et tâche effective (Leplat \& Hoc, 1983). Cependant, dans notre cas, les prescriptions ne portent pas seulement sur des types de tâches à réaliser, mais plus largement sur des dispositifs didactiques à mettre en place sur le terrain professionnel pour favoriser certains apprentissages. Nous proposons donc de distinguer le concept de système didactique prescrit, que nous utilisons comme un outil de caractérisation des dispositifs prescrits par l'institution école et celui de système didactique effectif, qui nous sert à étudier les processus de transmission de connaissances tels qu'ils se passent réellement au sein de l'institution entreprise. Reste encore à distinguer ces systèmes didactiques effectifs de leurs effets sur les apprentissages de l'apprenti. Cette dernière distinction est classique en didactique entre enseignement (on parle ici plus volontiers de transmission de connaissances) et apprentissage (Tiberghien, 1997).

\section{Méthodologie de constitution et d'analyse du corpus}

Le corpus sur lequel nous avons travaillé a été constitué à l'occasion d'une thèse sur le rôle des situations professionnelles dans la formation en alternance d'ingénieurs de production (Veillard, 2000, 2004). Il comporte des documents pédagogiques, des entretiens avec les formateurs et des notes d'observations au sein de l'école qui nous ont permis de comprendre l'organisation de 
l'alternance mise en place. Mais pour l'essentiel, il est constitué de différentes sources alimentant deux études de cas réalisées sur l'activité de deux apprentis ingénieurs en contexte professionnel. Le tableau 1 résume les caractéristiques principales de ces deux cas. Les entreprises ont été sélectionnées pour pouvoir comparer deux contextes organisationnels différents.

\begin{tabular}{|c|c|c|}
\hline & Cas 1 (Apprenti 1) & Cas 2 (Apprenti 2) \\
\hline Profil & 22 ans; BTS papeterie. & 21 ans ; DUT Génie Mécanique. \\
\hline Entreprise & $\begin{array}{l}\text { - Fabrication de papiers spéciaux haut de gamme (dessin, } \\
\text { emballages de luxe, etc.) } \\
-658 \text { personnes répartis sur } 4 \text { sites de production implantés } \\
\text { dans une même région en France. Majorité de personnel de } \\
\text { production. Ancienneté moyenne assez élevée. } \\
\text { - Grandes installations industrielles organisées selon un } \\
\text { process continu ; équipes de production en } 3 \times 8 \text { ou } 5 \times 8 \text {, } \\
\text { composées quasi exclusivement d'hommes }\end{array}$ & $\begin{array}{l}\text { - Fabrication de réactifs (ampoules ou plaques alvéolées) pour les } \\
\text { laboratoires d'analyse biologiques } \\
-3163 \text { salariés répartis sur } 4 \text { sites (A, B, C, D) implantés sur une } \\
\text { même région en France. Part très importante d'ingénieurs, cadres } \\
\text { et techniciens. Ancienneté moyenne faible. } \\
\text { - Petits ateliers organisés selon un process discontinu ; } \\
\text { Fonctionnement des équipes de production en } 2 \times 8 \text { (grande majorité } \\
\text { de femmes) }\end{array}$ \\
\hline $\begin{array}{l}\text { Mission et } \\
\text { rattachement }\end{array}$ & $\begin{array}{l}\text { Insertion dans le service de production regroupant } 3 \text { sites de } \\
\text { fabrication et les services techniques supports (bureau des } \\
\text { méthodes, service maintenance, etc.). } \\
\text { Projet: Amélioration de la propreté des papiers fabriqués } \\
\text { (mise en place de procédures et dispositifs techniques de } \\
\text { contrôle; essais de produits chimiques adjuvants; } \\
\text { sensibilisation des personnels de production). }\end{array}$ & $\begin{array}{l}\text { Insertion dans une antenne locale du département engineering (sur } \\
\text { le site B), service chargé de la mise en place des nouveaux } \\
\text { équipements techniques et de l'amélioration de l'organisation de la } \\
\text { production sur les différents sites. } \\
\text { Projet: Mise en place de } 3 \text { nouveaux équipements techniques de } \\
\text { conditionnement des produits et amélioration de l'organisation d'un } \\
\text { atelier. }\end{array}$ \\
\hline $\begin{array}{l}\text { Maître } \\
\text { d'Apprent. }\end{array}$ & $\begin{array}{l}\text { Diplômé d'une école de papeterie (grande école } \\
\text { d'ingénieur). Responsable du service production. }\end{array}$ & $\begin{array}{l}\text { Deux maîtres d'apprentissage successifs ( } 1 \text { an / 2ans). Le second est } \\
\text { diplômé du CESI (formation continue d'ingénieur en alternance); } \\
\text { Il est ingénieur méthodes, responsable local du service bureau } \\
\text { d'études sur le site B. }\end{array}$ \\
\hline Tuteur école & $\begin{array}{l}\text { Diplômé d'une grande école d'ingénieur généraliste; } \\
\text { nombreuses années de travail dans l'industrie sidérurgique. }\end{array}$ & $\begin{array}{l}\text { Agrégé en mécanique; enseignant précédemment dans une école } \\
\text { d'ingénieur en génie mécanique. }\end{array}$ \\
\hline
\end{tabular}

Tableau 1 - Principales caractéristiques des cas étudiés

Pour chaque apprenti, nous avons combiné deux niveaux de description de son activité en entreprise et de son contexte de réalisation. Le premier niveau est une description à grande échelle qui couvre les trois années de formation. Pour le constituer, nous avons croisé et recoupé plusieurs sources: des entretiens avec l'apprenti, le tuteur entreprise et le tuteur école; des documents pédagogiques; des archive dans l'entreprise. Le deuxième niveau est une description beaucoup plus fine, réalisée sur une durée de huit jours, au milieu de la deuxième année de formation. Pour la constituer, nous nous sommes basés sur des notes d'observation sur le terrain, complétées par des enregistrements sonores des conversations de l'apprenti. Puis nous avons procédé à une auto-confrontation (Theureau, 1991) de l'apprenti à cette première description pour l'enrichir et la corriger.

L'analyse du corpus est menée en deux temps. Tout d'abord, nous étudions a priori, à l'aide du concept de système didactique prescrit, l'organisation du parcours en entreprise planifiée par l'institution scolaire (partie 3 de 1'article). Puis, à partir des deux études de cas, nous tentons de caractériser les différents systèmes didactiques réels qui ont jalonné le parcours des deux apprentis dans leur entreprise pendant trois ans, pour finalement les mettre en lien d'une part avec les systèmes didactiques prescrits et d'autre part avec l'activité et les apprentissages de l'apprenti. 


\section{L'organisation mise en place par l'école}

\subsection{Organisation générale de l'alternance}

L'objectif général de l'école est de former des ingénieurs de production en alternance, sous contrat d'apprentissage. Cet établissement privé, né dans les années 90, recrute essentiellement des étudiants titulaires d'un Brevet de Technicien Supérieur (BTS) ou d'un Diplôme Universitaire de Technologie (DUT) de spécialités techniques.

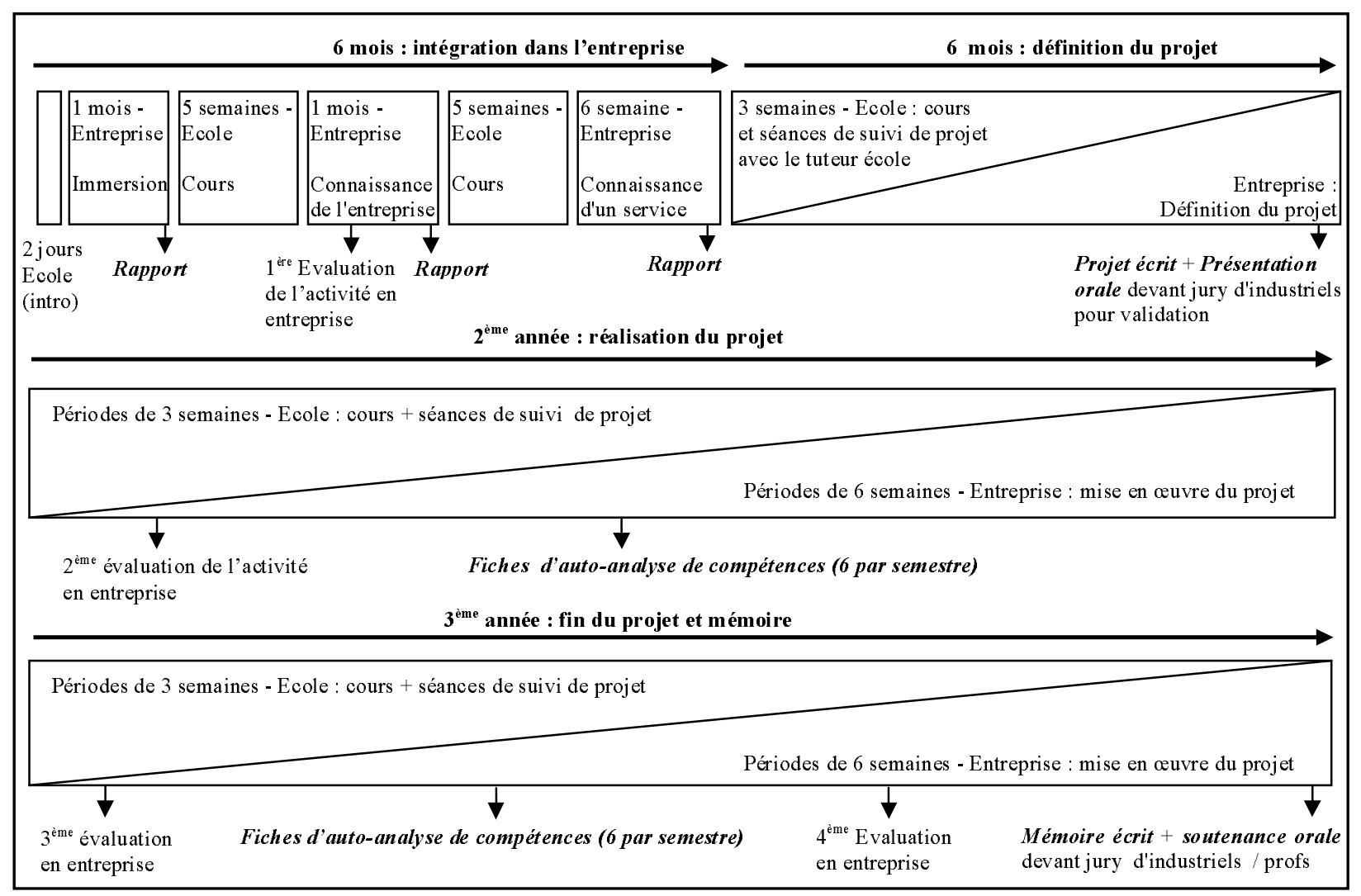

Figure 1 : Organisation générale de la formation

La formation s'appuie sur un référentiel qui décrit le profil de l'ingénieur à former, caractérisé par une double compétence : être capable de gérer quotidiennement une unité de production dans ses dimensions techniques, économiques et humaines; apporter des améliorations à cette unité sur ses différentes dimensions afin d'assurer son évolution et sa pérennité à travers une démarche de gestion de projet industriel. Les enseignements à l'école et l'activité au sein d'une entreprise sont censés participer de manière complémentaire à l'atteinte de cet objectif général. La figure 1 donne une vue schématique de l'organisation pédagogique centrée sur le parcours en entreprise. Trois grandes étapes scandent ce parcours : la période d'intégration (six premiers mois); la définition du projet (six mois suivants); la réalisation du projet et le mémoire (deuxième et troisième année. Chaque apprenti est encadré dans l'entreprise par un tuteur entreprise et suivi par un tuteur de l'école. 


\subsection{Analyse des systèmes didactiques prescrits}

L'analyse de l'organisation du parcours en entreprise nous a conduit à dégager six types de systèmes didactiques prescrits, synthétisés dans le tableau 2. L'impression qui se dégage à la lecture du tableau est à la fois l'ampleur temporelle du cadrage tenté par l'école (les trois années de la formation) et la complexité du dispositif mis en place, due en particulier à l'entrecroisement d'acteur et d'objets issus des deux types d'institution au sein des différents systèmes didactiques prescrits.

Ainsi, dans tous ces systèmes, il doit y avoir action coordonnée d'au moins deux types d'acteurs de deux institutions différentes au sein du pôle enseignant, là où, dans les situations scolaires, un seul enseignant de la même institution occupe généralement cette position. En ce sens, le système didactique de type 2 constitue un extrême puisque d'autres acteurs de l'entreprise doivent aussi participer au pôle enseignant. On peut donc imaginer que la communication et la coordination entre ces différents acteurs va être primordiale pour que ces systèmes didactiques prescrits puissent être mis en oeuvre.

Le milieu de l'apprenti doit aussi se composer de différents objets types provenant des deux institutions. Le schéma le plus fréquent est le suivant: des objets de l'école (questionnement, concepts, grilles d'analyse) doivent être utilisés comme des outils d'orientation de 1'activité de l'apprenti et d'étude des objets (dispositifs techniques, relations humaines, etc.) de l'institution entreprise. Il en est ainsi, par exemple, pour l'étude d'un service ( $3^{\text {ème }}$ période), qui demande de considérer successivement l'organisation selon des logiques hiérarchique, fonctionnelle et processus.

Autre point remarquable : les objets du milieu sont à considérer sous de multiple rapports. En particulier, une distinction peut être faite entre les rapports d'étude, dont la finalité principale est de connaître et comprendre les caractéristiques de certains objets de l'entreprise (systèmes didactiques de type 2 et 5 ) et des rapports à finalité productive, où l'apprentissage est un corollaire de 1'action. Lorsque cette formation a été créée en 1992, le début du parcours en entreprise des apprentis était beaucoup moins cadré : les systèmes didactiques prescrit de type 1 , 2 et 5 n'existaient pas et les étudiants étaient censés travailler d'emblée à la définition de leur projet. Mais rapidement, les responsables de la formation ont été contraints de revoir cette organisation, suite à des difficultés importantes des apprentis à trouver leurs repères au début du parcours en entreprise et à prendre du recul à l'occasion du mémoire de fin d'études. Ils ont alors imaginé de consacrer les six premiers mois du parcours en entreprise à une étude de son organisation à différents niveaux (systèmes didactiques 1 et 2 ) et mettre en place, en fin de formation, un dispositif d'auto-analyse des compétences pour amener les apprentis à devenir plus réflexifs (système didactique 5)

La variété des types de rapports à finalité productive est également frappante : rapports d'usage de dispositifs techniques, d'autorité dans le management des hommes, d'analyse des performances des hommes et des machines, de création de nouvelles organisations, etc. 
Tableau 2 - Caractéristiques des différents types de systèmes didactiques prescrits

\begin{tabular}{|c|c|c|c|c|c|}
\hline SD & $\begin{array}{l}\text { Position dans } \\
\text { la formation }\end{array}$ & Enjeu(x) didactique(s) & $\begin{array}{c}\begin{array}{c}\text { Pôle enseignant (qui et quelles } \\
\text { responsabilités didactiques) }\end{array} \\
\end{array}$ & \begin{tabular}{|c|}
$\begin{array}{c}\text { Milieu (objets et types de rapports } \\
\text { dominants) }\end{array}$ \\
\end{tabular} & Responsabilités de l'apprenti \\
\hline Type 1 & $\begin{array}{l}\text { Période } \\
d^{\prime} i m \text { mersion } \\
\left(1^{\text {er }} \text { mois }\right)\end{array}$ & $\begin{array}{l}\text { - Connaître / Expérimenter ce } \\
\text { qu'est le travail ouvrier et le } \\
\text { contexte d'un atelier }\end{array}$ & $\begin{array}{l}\text { - Tuteur entreprise : introduire l'apprenti } \\
\text { dans l'atelier et expliquer son statut aux } \\
\text { ouvriers } \\
\text {-Ouvriers (expérimentés) : intégrer } \\
\text { 1'apprenti / lui apprendre un poste de travail / } \\
\text { lui donner des tâches }\end{array}$ & $\begin{array}{l}\text { - Objets : dispositifs techniques et équipes de } \\
\text { production ; } \\
\text { - Rapports : usage (appareils techniques) } \\
\text { autorité et transmission (ouvriers expérimentés, } \\
\text { chefs d'équipes), coopération (autres ouvriers) }\end{array}$ & $\begin{array}{l}\text { - Tenir un poste d'exécution de bas } \\
\text { niveau } \\
\text { - Se plier aux prescriptions et } \\
\text { explications des ouvriers }\end{array}$ \\
\hline Type 2 & $\begin{array}{l}\text { Périodes } \\
\text { d'immersion, } \\
\text { connaissance de } \\
\text { l'entreprise } \\
\text { connaissance } \\
\text { d'un service }\end{array}$ & $\begin{array}{l}\text { - Connaître l'organisation générale } \\
\text { de l'entreprise } \\
\text { et l'organisation de la production }\end{array}$ & $\begin{array}{l}\text { - Tuteur école : expliquer les attentes de } \\
\text { 1'école, la méthode à utiliser; évaluer la } \\
\text { compréhension de l'organisation } \\
\text { - Tuteur entreprise : donner accès aux } \\
\text { ressources nécessaires aux rapports } \\
\text { - Autres acteurs : transmettre les } \\
\text { informations et connaissances sur } \\
\text { 1'organisation des services, de la production, } \\
\text { etc. }\end{array}$ & $\begin{array}{l}\text { - Objets : organigrammes fonctionnels et } \\
\text { hiérarchiques; procédures / manuels qualité ; } \\
\text { résultats et politique de l'entreprise ; différents } \\
\text { services et acteurs ; prescriptions de l'école ; } \\
\text { grilles d'analyse } \\
\text { - Rapports : étude (pas d'enjeu productif, juste } \\
\text { apprendre) }\end{array}$ & $\begin{array}{l}\text { - Aller dans différents services pour } \\
\text { trouver les informations et les } \\
\text { connaissances et interroger les acteurs } \\
\text { - Utiliser le plan et les méthodes } \\
\text { fournies par l'école (ex : méthodes } \\
\text { d'analyse fonctionnelle, par processus, } \\
\text { hiérarchique) } \\
\text { - Rédiger les rapports selon le plan } \\
\text { demandé par l'école }\end{array}$ \\
\hline Type 3 & $\begin{array}{l}\text { Phase } \\
\text { d'élaboration du } \\
\text { projet }\end{array}$ & $\begin{array}{l}\text { - Apprendre à analyser les } \\
\text { performances d'un système } \\
\text { productif } \\
\text { - Connaître les différentes } \\
\text { dimensions d'un projet d'ingénieur } \\
\text { et savoir concevoir un tel projet } \\
\text { - Construire un contexte } \\
\text { d'apprentissage du management de } \\
\text { projet }\end{array}$ & $\begin{array}{l}\text { - Tuteur école : expliquer les attendus sur le } \\
\text { projet ; expliquer la méthode de } \\
\text { questionnement ; évaluer l'ampleur du projet } \\
\text { - Tuteur entreprise : donner un thème de } \\
\text { projet profitable à l'entreprise ; aider et } \\
\text { former à l'analyse du système productif; } \\
\text { valider la pertinence du projet pour } \\
\text { l'entreprise }\end{array}$ & $\begin{array}{l}\text { - Objets : dispositifs techniques, équipes, données } \\
\text { de production des ateliers; ; services techniques } \\
\text { (méthodes; etc.); concepts et méthodes } \\
\text { d'ingénierie et de gestion de projet ; grille de } \\
\text { questionnement de l'école } \\
\text { - Rapports : analyse (diagnostic des } \\
\text { performances et des problèmes; coopération } \\
\text { (ingénieurs, techniciens, fournisseurs); création } \\
\text { (nouvelle organisation productive); }\end{array}$ & $\begin{array}{l}\text { - Solliciter le tuteur Entreprise pour } \\
\text { définir un thème de projet } \\
\text { - Utiliser la grille de questionnement } \\
\text { fournie } \\
\text { - Utiliser les concepts et méthodes } \\
\text { d'ingénierie pour le diagnostic et la } \\
\text { conception du projet }\end{array}$ \\
\hline Type 4 & $\begin{array}{l}\text { Phase de } \\
\text { conduite du } \\
\text { projet }\end{array}$ & $\begin{array}{l}\text { - Apprendre à manager un projet } \\
\text { sur différents aspects notamment } \\
\text { management du changement } \\
\text { auprès des équipes de production }\end{array}$ & $\begin{array}{l}\text { - Tuteur entreprise : pousser à la prise de } \\
\text { responsabilités ; transmettre son expérience ; } \\
\text { valider les décisions dans le cadre du projet ; } \\
\text { évaluer les actions et le comportement } \\
\text { - Tuteur école : expliquer le dispositif } \\
\text { d'évaluation au tuteur entreprise et à } \\
\text { l'apprenti }\end{array}$ & $\begin{array}{l}\text { - Objets : dispositifs et équipes de production; } \\
\text { services techniques ; fournisseurs de l'entreprise ; } \\
\text { méthodes de management et de gestion de projet ; } \\
\text { référentiel métier de l'école } \\
\text { - Rapports : analyse; } \\
\text { autorité (management de l'équipe projet ; des } \\
\text { acteurs de l'atelier) }\end{array}$ & $\begin{array}{l}\text { - Prendre en main rapidement } \\
\text { l'ensemble du projet ; } \\
\text { - prendre le référentiel métier comme } \\
\text { repère pour l'action d'ingénieur } \\
\text { - Utiliser des outils de gestion de } \\
\text { projet (planification, budget, fiches } \\
\text { bilans, etc.) }\end{array}$ \\
\hline Type 5 & $\begin{array}{l}\text { Phase de } \\
\text { conduite du } \\
\text { projet }\end{array}$ & $\begin{array}{l}\text { - Apprendre à être réflexif sur ses } \\
\text { propres actions, les résultats } \\
\text { obtenus et inférer à partir de là des } \\
\text { compétences acquises }\end{array}$ & $\begin{array}{l}\text { - Tuteur école : expliquer les attentes et } \\
\text { outils proposés par l'école; pousser à la } \\
\text { prise de recul sur les actions; favoriser la } \\
\text { comparaison entre apprentis; } \\
\text { - Tuteur entreprise : valider les compétences } \\
\text { décrites }\end{array}$ & $\begin{array}{l}\text { - Objets : ses actions dans l'entreprise } \\
\text { - Rapports : étude (pas d'enjeu productif } \rightarrow \\
\text { développer la réflexivité) }\end{array}$ & $\begin{array}{l}\text { - Prendre du temps pour écrire son } \\
\text { expérience } \\
\text { - Utiliser les grilles fournies par } \\
\text { l'école pour analyser ses compétences } \\
\text { - Préparer des présentations orales sur } \\
\text { le projet ; }\end{array}$ \\
\hline Туре 6 & Fin du projet & $\begin{array}{l}\text { - Apprendre à faire le bilan d'un } \\
\text { projet : évaluation de la méthode et } \\
\text { de ses effets par rapport aux } \\
\text { attendus }\end{array}$ & $\begin{array}{l}\text { - Tuteur école : expliquer les attendus du } \\
\text { jury; aider à la création du plan et la } \\
\text { rédaction } \\
\text { - Tuteur entreprise : aider à l'analyse du } \\
\text { projet en lien avec la stratégie, le bilan des } \\
\text { effets du projet, etc. }\end{array}$ & $\begin{array}{l}\text { Objets : comptes-rendus de projet; stratégie } \\
\text { économique de l'entreprise ; performances du } \\
\text { système productif après projet ; ses actions dans } \\
1 \text { 'entreprise } \\
\text { Rapports : analyse (bilan du projet) ; étude (pas } \\
\text { d'enjeu productif : développer la réflexivité sur } \\
\text { 1'action) }\end{array}$ & $\begin{array}{l}\text { - S'appuyer sur les archives du projet } \\
\text { - Respecter les consignes de l'école } \\
\text { (plan type, style d'écriture, domaines } \\
\text { abordés, date de rendu) }\end{array}$ \\
\hline
\end{tabular}


Cette variété est à mettre en relation avec le métier d'ingénieur de production, un ingénieur terrain qui se distingue assez nettement de la figure de l'ingénieur spécialiste d'un domaine technique précis, à la fois par la variété beaucoup plus grande des objets qu'il doit prendre en compte (dispositifs techniques, personnel de production, méthodes de gestion de production, de management, de gestion de projet, etc.) et par les types de rapport variés à construire avec ces objets (autorité dans le management quotidien, analyse et création dans la gestion de projet, etc.)

Notons enfin que l'explicitation des contrats didactiques censés réguler ces différents systèmes didactiques est très variable. Ainsi, lors des six premiers mois, les attentes de l'institution école sont relativement précises quant à ce que doivent faire l'apprenti et les deux tuteurs. Par la suite, on attend plus vaguement que l'apprenti définisse et gère un projet industriel sur deux ans. Le tuteur entreprise dispose alors d'une grande liberté pour encadrer l'apprenti, l'école insistant seulement sur l'importance d'une action vigoureuse pour pousser les apprentis à prendre toutes les responsabilités attendues. Pour le reste, les prescriptions scolaires sont en retrait, les demandes venant après l'action, pour tenter d'exploiter réflexivement l'expérience développée.

\section{Première étude de cas (entreprise papetière)}

\subsection{Les six premiers mois d'intégration}

Le tuteur entreprise est convaincu du bien-fondé de l'organisation proposée par l'école. Au cours d'un entretien, il insiste beaucoup sur l'importance de la période d'immersion dans les ateliers.

MA1 : «Sa première mission, c'était de connaître l'entreprise, et je pense qu'il y a toujours un intérêt à la connaître par le terrain [...] Je voulais qu'il vive le coté opérationnel, le coté opérateur terrain parce que une fois qu'on est de l'autre coté de la barrière une fois qu'on est dans des rôles d'encadrant [...] on est confronté à des situations, il faut savoir les appréhender et il vaut mieux avoir vécu les choses et comprendre comment peut réagir un opérateur» (Entretien tuteur entreprise 1).

Cette forte conviction le conduit à plonger l'apprenti dans les trois ateliers de fabrication pendant sept semaines au lieu des quatre demandées par l'école. Selon lui, c'est le temps nécessaire pour connaître suffisamment les installations techniques et les personnes de ces services. Dans chaque atelier, il se déplace pour le présenter aux responsables. Ceux-ci le positionnent comme aidemachine, un travail de manutention et de nettoyage, qui correspond au plus bas niveau dans l'échelle des postes en production mais constitue le passage obligé de tout nouvel ouvrier. Notre apprenti se trouve immergé dans un premier type d'institution, où de nombreux systèmes didactiques effectifs se mettent en place entre les ouvriers expérimentés et lui-même. A cette occasion, on lui transmet non seulement des techniques de travail, mais aussi des représentations sur l'entreprise et ses responsables (par exemple le bien-fondé des choix en matière de flexibilité de production). L'apprenti crée des rapports avec les ouvriers basés sur un fort respect de leur vision de la production et de leur autorité. Le tuteur entreprise lui reproche d'ailleurs ce trop grand respect, lors de la première évaluation en entreprise, comme en témoigne cette appréciation rédigée dans son livret d'évaluation.

«A visiblement du mal à s'intégrer dans les équipes, devrait être plus volontariste et moteur. Semble être motivé au travers des contacts avec l'encadrement, mais cette motivation semble disparaittre auprès des opérateurs. Devrait profiter de ses connaissances pour se faire valoir auprès des opérateurs : suggestion, aide, etc. » (Extrait du livret d'évaluation de l'apprenti 1) 
Les systèmes didactiques prescrits lors de la deuxième et troisième période en entreprise (type 2) se mettent effectivement en place, conformément au souhait de l'école. Le tuteur entreprise joue son rôle en l'introduisant auprès des interlocuteurs à interroger et lui laisse tout le temps nécessaire pour effectuer ce travail. L'apprenti étudie différents documents, notamment les procédures qualité qui sont une source privilégiée, interroge différentes personnes au sein des services qui se plient de bonne grâce à l'exercice, observe longuement les installations techniques et l'organisation du travail et finalement rédige les rapports conformément au cahier des charges fixé par l'école. Le fait qu'il connaisse déjà l'univers technique de la papeterie grâce à son BTS, l'aide beaucoup à comprendre l'organisation technique de la production.

\section{La phase de définition du projet}

Le tuteur entreprise souhaite que le projet porte sur l'amélioration de la propreté des papiers produits, dans un contexte économique où la concurrence s'accroît et la qualité de la production devient plus déterminante. Il décide de confier l'apprenti au soin d'un technicien du service Recherche et Développement $(\mathrm{R} \& D)$ à qui il a demandé depuis quelques mois de réaliser des analyses sur les causes de non-propreté des papiers. Ce faisant, il pense faire coup double: soulager ce technicien à qui l'on a demandé de faire ces analyses en plus de son travail habituel ; introduire l'apprenti à la problématique du projet et lui faire connaître les interlocuteurs, les moyens techniques et les règles organisationnelles dont 11 aura besoin.

Notre apprenti bascule alors dans un second type d'institution, où il se trouve d'abord en position d'assistant de ce technicien. De nombreux systèmes didactiques émergent dans le cours de l'action du technicien, qui transmet à cette occasion son savoir faire : les méthodes de collecte d'échantillons et d'interrogation des équipes de production ; les techniques d'analyse avec les outils du laboratoire (microscope, réactifs, protocoles d'analyse, etc.) ; les styles de compte-rendu à destination du responsable de production et des chefs d'atelier. Comme avec les ouvriers, l'apprenti créé un rapport de grand respect du savoir-faire de ce technicien et de sa vision de l'entreprise. Peu a peu, il devient autonome et prend les analyses de plus en plus à sa charge. Il apporte définitivement ses affaires dans les locaux de ce service (elles étaient jusque là dans le bureau des contremaîtres de 1'atelier 2) et s'installe dans la position du technicien jusqu'à le remplacer complètement.

$\mathrm{A} 1:$ : Petit à petit, j'ai pris sa place (Technicien R\&D) alors que normalement c'était pas du tout ça que je devais faire ». (Entretien apprenti 1)

Comme il dispose de temps entre ces analyses, l'apprenti prend l'initiative d'améliorer les procédures existantes et de les réorganiser dans des classeurs. Les acteurs de l'entreprise (équipes de production, services techniques) finissent par le reconnaître comme un technicien du service R\&D. Le tuteur entreprise intervient à deux reprises pour lui demander de réaliser des études techniques complémentaires en lien avec la problématique du projet. Mais son milieu change peu : il est principalement constitué d'objets du laboratoire R\&D et des ateliers de production, avec lesquels il a créé des rapports d'analyse.

La définition formelle du projet va se faire en parallèle. Poussé par le calendrier et le tuteur école, l'apprenti questionne son tuteur entreprise sur ce que pourrait être ce projet. En première 
intention, le tuteur entreprise lui propose de mettre en place un dispositif technique de contrôle optique de la propreté du papier. Mais les documents fournis par l'école (grille de questionnement, cahier des charges du projet), relayés par son apprenti anxieux de ne pas avoir un projet conforme aux prescriptions, le conduisent à élargir le projet à différents moyens (techniques, organisationnels, formation) pour améliorer la propreté du papier produit. Le document finalement rédigé est approuvé par la commission d'ingénieurs mise en place par l'école pour valider les projets. Moyennant quelques allers et retours entre les deux tuteurs, le système didactique prescrit pour la définition du projet est donc mis en œuvre conformément aux prescriptions de l'école.

\section{La réalisation du projet}

A ce stade, on devrait assister à une évolution progressive de la position de l'apprenti vers une prise en charge de plus en plus complète du projet. On constate au contraire une grande continuité dans son activité par rapport à l'étape précédente: les analyses des causes de problème de propreté, complétées par quelques études techniques, restent le gros de son quotidien. Le tuteur entreprise finit d'ailleurs par s'en inquiéter.

MA1 : " ça va pas vite, pour rentrer dans la peau d'un chef de projet, c'est vraiment dur pour ces gens là je pense qu'il faut généraliser s'il y a pas quelqu'un pour piloter de très près ça n'avance quasiment pas ou ça reste de l'étude de documents mais il ne va pas au contact de l'opérateur il ne va pas au devant des gens qui savent [...] c'est une phase où je pense l'étudiant apprenti a besoin d'être hyper piloté tout en étant secoué pour que ce soit lui qui prenne des initiatives » (entretien tuteur entreprise 1).

Il va alors tenter de le pousser vers ce rôle de chef de projet en lui donnant plusieurs petites missions successives telles que: organiser l'essai d'un nouveau produit dans les installations; inspecter la propreté des tuyauteries ; suivre la mise en place d'un système de contrôle optique de la propreté des papiers. Nous analysons ces interventions du tuteur comme autant de tentatives pour mettre en place des systèmes didactiques censés apprendre progressivement à l'apprenti à gérer une action d'amélioration de l'appareil productif. Car il fait plus que donner du travail en mettant en place 1'organisation suivante : un rendez-vous pour expliquer à l'apprenti les objectifs attendus, puis des rencontres avec lui toutes les semaines ou tous les quinze jours pour évaluer l'avancement de l'action, le questionner sur ce qu'il a compris et lui donner des conseils sur la marche à suivre. Malgré ces tentatives, l'apprenti rechigne à endosser les habits d'un manager de projets et continue à privilégier les études techniques, souvent très pointues, d'analyse des causes de non propreté. La transition vers la position de manager de projet apparaît alors comme freinée, voire empêchée, par des rapports à des objets non adaptés à cette nouvelle position.

Les rapports aux ouvriers. Lors de nos observations sur le terrain, nous avons capté plusieurs échanges dans l'atelier qui montrent que l'apprenti a du mal à dépasser le rapport aux ouvriers élaboré lors de la période d'immersion. L'extrait ci-dessous arrive après un moment de tension, où un essai d'adjuvant chimique censé améliorer la propreté du papier a produit l'effet inverse. Le tuteur entreprise vient de quitter les lieux, après avoir donné des consignes aux opérateurs pour poursuivre l'essai sous certaines conditions. Ces derniers (Op1, Op2) se plaignent auprès de l'apprenti (A1) contre la flexibilité accrue de la production qui, selon eux, est une des causes importantes des problèmes de propreté. 
Op1 : un coup on a fait de la fibre rouge et on a enchaîné derrière [sur du papier blanc] / on faisait du papier rose

Op2 : alors ça c'est encore plus con

A2: Du papier rose ouais

Op1 : on a fait après, on a fait je sais pas combien de cassés [papier qui déchire sur la machine et qu'il faut jeter au rebut] on a fait/deux ou trois tonnes de cassés

Op2 : oh ce merdier

A2: ouais on n'a pas vraiment nettoyé ce qui comptait c'était la production

Op1 : la production, j'aime bien la production / quand tu vois des papiers comme ça là [i1 montre un échantillon de papier sale] qui sont aussi dégueulasses / la production /

$\mathrm{A} 2$ : il faut trouver un compromis entre la production et la qualité quoi

Op1 : il faut savoir ce qu'on veut quoi / avant on produisait peut-être moins mais on faisait de la qualité quand même / tu faisais un lavage des fois / on s'est eu payé des lavages $6 \mathrm{~h}$

A2 : alors que maintenant c'est $2 h$

Op1 : et on n'avait pas les circuits qu'on a maintenant/maintenant on a des circuits qui sont en inox et tout.

Op2: maintenant quand tu laves t'essaies de le faire comme y faut tu te fais engueuler parce que tu as mis trop de temps /

A2 : ouais, ils essaient de combler la capacité de la machine par des arrêts moins longs en fait

Op 1 : ouais c'est pas une solution

$\mathrm{A} 2$ : bein non

Là où il faudrait se positionner du côté de l'encadrement et du tuteur entreprise et justifier les choix de flexibilité accrue, 1'apprenti approuve très vite les opérateurs. Son utilisation du «ils» pour désigner la direction du service et de l'entreprise indique clairement de quel côté il se positionne, comme le confirment ses propres propos lors de l'auto-confrontation :

" [les ouvriers)] me considèrent plus comme un mec de la machine que comme un futur ingénieur on délirait avec eux ». (auto-confrontation, apprenti 1)

Les rapports d'analyse aux objets de l'atelier. Quand il faut prendre des décisions sous la contrainte, situation typique de celle d'un manager, l'apprenti n'arrive pas à risquer une réponse, et se réfugie derrière la nécessité de faire des analyses, même lorsque le tuteur entreprise le met plus directement à l'épreuve. Il demeure dans des rapports d'analyse distanciée aux objets et acteurs des ateliers, tels qu'il a pu les créer au sein du laboratoire R\&D dans sa position de technicien.

Le rapport au tuteur entreprise et aux managers. L'apprenti n'a jamais pu partager le quotidien du tuteur entreprise, ni celui d'un autre responsable de service ou de projet, ce qui n'a pas permis l'émergence de systèmes didactiques dédiés à la transmission des connaissances de ce type. Il n'est donc pas surprenant que l'apprenti ait une faible connaissance des circuits de décision dans l'entreprise. Les décisions importantes et la coordination effective du projet sont assurées par le tuteur entreprise ou d'autres acteurs, sans d'ailleurs que l'apprenti soit toujours bien informé de l'évolution des choses. Ainsi, en ce qui concerne la mise en place du système de contrôle optique de la propreté des papiers, l'apprenti apparaît clairement hors jeu du management.

TE1 : je n'avais pas franchi dans ma tête l'étape qui permettait de dire il est intéressant pour l'entreprise d'investir dans ce genre de matériel. Je me souviens l'avoir franchi début 97 [...] Cette décision je l'ai prise seule $[\ldots]$ 
Chercheur : qui contacte les fournisseurs dans ce cas?

TE1 : j'ai confié ça à $R[\ldots]$ c'est notre homme des automatismes, de l'électronique. C'est lui qui a été nommé responsable du projet achat et mise en place d'une caméra » (entretien tuteur entreprise 1).

Il faut noter cependant une petite évolution en fin de formation, lorsque, par exemple, l'apprenti demande à participer aux réunions de production pour pouvoir informer plus efficacement les responsables des ateliers sur l'évolution des problèmes de propreté.

Finalement, alors que notre analyse met en évidence qu'il ne termine pas dans la position formellement prescrite par l'institution école, celle-ci semble pourtant satisfaite de son évolution. Ainsi, le tuteur école juge ses fiches d'analyse de compétences de bonne qualité et minore le jugement plus sévère du tuteur entreprise quand celui-ci lui reproche son manque de leadership et de prise en main du projet. A la fin de l'année, le jury du mémoire le félicite pour la qualité du travail effectué et son recul sur la réalisation du projet. L'apprenti a bien compris l'intérêt de jouer le jeu des deux derniers systèmes didactiques prescrits (type 5 et 6 ) : ils lui permettent de développer un bon recul réflexif sur ses actions et de bien comprendre la cohérence d'ensemble du projet et son importance stratégique pour l'entreprise. Grâce à cela, et malgré une implication faible sur le terrain du management, il parvient à valoriser fortement son travail auprès de l'école, en jouant notamment sur l'utilisation de pronoms impersonnels qui masquent ses responsabilités réelles.

\section{Seconde étude de cas}

\subsection{Les six premiers mois d'intégration}

Dans ce deuxième cas, l'apprenti ne fait pas de période d'immersion dans un atelier. Il se trouve de suite en position de technicien méthodes au sein d'une institution (antenne locale du service engineering sur le site B) dans laquelle il restera jusqu'à la fin de la formation et dont la finalité est de gérer des projets industriels. Manquant de moyens humains, le tuteur entreprise a besoin de lui immédiatement pour aider à la mise en place des projets d'automatisation de la production prévus sur le site. Après lui avoir laissé deux jours pour étudier l'organisation de l'entreprise, du site et du service à partir de documents (plaquette de présentation et manuel de procédures qualité), il lui confie un travail de conception et de mise en place de pièces techniques sur une nouvelle machine de conditionnement des produits qui se trouve en phase de test dans un des ateliers. Très vite, l'apprenti doit être autonome car son tuteur est très occupé et le renvoie vers d'autres personnes quand il le sollicite. De multiples systèmes didactiques, d'existence parfois très courte se mettent en place avec des personnes du service engineering mais aussi d'autres services. Par exemple, il sollicite plusieurs personnes ponctuellement (un technicien du service, un apprenti d'une autre formation, des techniciens de la maintenance) pour apprendre à utiliser un logiciel de dessin technique nécessaire à la conception de pièces. Il tisse ainsi rapidement un réseau important d'interlocuteurs experts dans l'entreprise

A2 : "on me consultait. J'avais une petite partie à faire et on me demandait où ça en était. J'ai connu tout le monde très rapidement [..] parce que j'étais tout le temps à droite à gauche (entretien apprenti 2).

Il passe aussi beaucoup de temps à observer la machine dans l'atelier pour comprendre son fonctionnement et prendre les mesures des pièces à concevoir. Enfin, il contacte de manière 
autonome plusieurs fournisseurs pour la réalisation technique des pièces et assure lui-même le suivi de leur fabrication et de leur mise en place dans l'atelier. Le tuteur entreprise intervient ponctuellement pour surveiller son travail, lui donner quelques conseils techniques et valider les choix les plus importants, par exemple les caractéristiques des pièces et le fournisseur qui les fabriquera.

Les systèmes didactiques prescrits par l'école, visant l'étude de l'entreprise (type 2) sont faiblement relayés sur le terrain par le tuteur entreprise.

$\mathrm{A} 2$ : «Les rapports je les ai tous fait la dernière semaine, voire chez moi après donc / sachant que [...] $C$ [tuteur entreprise] n'était pas trop chaud pour que fasse les rapports quand j'étais dans l'entreprise " (entretien apprenti 2).

Pris par l'urgence, l'apprenti recopie in extenso des documents trouvés dans l'entreprise et répond très partiellement aux cahiers des charges fixés par l'école. Malgré les critiques du tuteur école, l'apprenti considère ces demandes comme secondaires, même s'il reconnaît qu'elles lui ont permis de mieux comprendre l'organisation du processus de production. Le tuteur entreprise joue si peu le jeu demandé par l'école que la première évaluation en entreprise se traduit par un raté : le tuteur école attend en vain que le tuteur entreprise finisse une réunion pour pouvoir le recevoir. Il faudra plusieurs relances pour que cette première évaluation se tienne finalement, mais dans les locaux de l'école et non de l'entreprise comme c'est normalement le cas.

\subsection{La définition du projet}

A ce stade, rien ne change véritablement par rapport à la première étape. L'apprenti termine la mise en place des pièces qu'il a conçues, puis participe activement aux tests d'utilisation de cette nouvelle machine par les opérateurs. Parallèlement, le tuteur entreprise lui confie une tâche similaire de conception de pièces sur une autre machine. L'apprenti démarre ce travail aussitôt.

La phase de définition du projet demandé par l'école est très chaotique. Suite à des relances répétées de l'apprenti, lui-même mis sous pression par le timing imposé par l'école, le tuteur entreprise finit par lui répondre que le projet consistera à terminer les tests sur la machine de conditionnement, puis à suivre sa mise en production réelle et faire un suivi des flux au sein de l'atelier. L'apprenti rédige ses premières fiches de présentation du projet sur cette base, mais ce projet est jugé trop étriqué par le tuteur école (Tec2).

TEc2: «Je me rappelle que là, c'était bloquant parce que lui s'attardait uniquement à décrire ce petit projet et on avait pas une vision de toute la formation [...] Il était sur 6 mois, à peu près 6-8 mois, c'était un petit truc » (entretien tuteur école 2)

L'apprenti sollicite à nouveau le tuteur entreprise mais celui-ci élude plusieurs fois les questions, repoussant à plus tard la définition de ce projet. Il a du mal à lui proposer un projet conforme aux attentes de l'école, car la règle dans l'institution veut que ceux-ci ne soient confiés qu'à des ingénieurs expérimentés.

Peu de temps après, il annonce à l'apprenti qu'il va bientôt changer de fonction et quitter le site B. Un autre ingénieur du service se déclare alors intéressé par la reprise du tutorat, ayant constaté 
les qualités d'autonomie du jeune homme. Durant l'été, il rencontre le tuteur école et l'apprenti pour définir le projet. Pour se sortir de l'impasse due à la règle de ne pas confier de gros projets à des novices, les deux encadrants vont mettre au point un système didactique de compromis : il s'agit d'une combinaison de plusieurs petits projets séquencés selon une logique de progression de l'activité de 1'apprenti (cf. figure 2). Il s'agit bien là d'un artefact didactique, car la séquentialisation de ces projets répond à des contraintes liées à l'apprentissage : la complexité globale est anticipée comme croissante en raison d'une charge de travail et de responsabilités progressives et de la nécessité de coordonner de plus en plus d'actions simultanément. Il s'agit de favoriser autant que possible l'autonomie de l'apprenti, le tuteur entreprise devant intervenir ponctuellement pour valider les choix et donner quelques recommandations sur la manière de faire.

Ce cadre didactique n'est pas élaboré via la grille de questionnement, comme l'a bien compris 1'apprenti.

\section{A2 : "On partait du principe que le questionnement était là pour t'aider à rédiger ton avant-projet [...]}

Sauf que moi j'ai fait l'avant-projet sans utiliser le questionnement »(entretien apprenti 2)

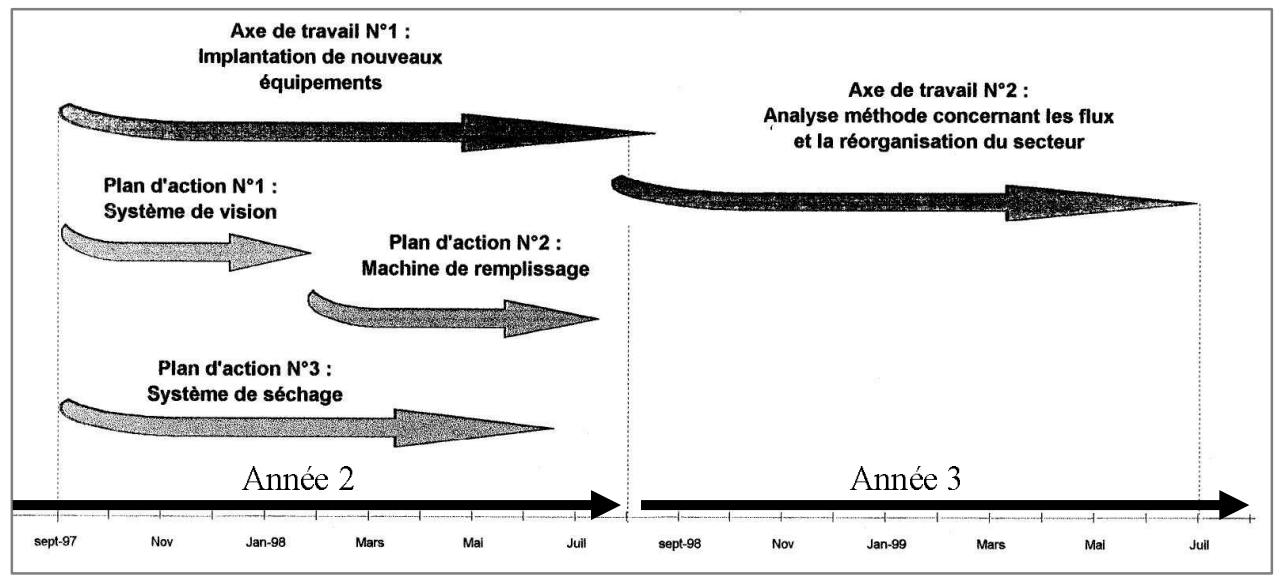

Figure 2 : Echelonnement des projets successifs (tiré du Projet de l'élève).

Il n'est donc pas exagéré de dire que le système didactique prescrit de type 3 est peu pris en compte tout au long de cette seconde étape du parcours en entreprise.

\subsection{La mise en œuvre du projet}

La troisième étape se traduit par la réalisation des projets définis sans évènements très marquants. La progression de l'apprenti se passe comme prévue, sans difficultés particulières, y compris lorsqu'il lui faut convaincre les équipes de production de modifier leurs habitudes. Il contribue de lui-même à l'émergence de nombreux systèmes didactiques, parfois très ponctuels, en sollicitant des personnes expertes qui lui transmettent des connaissances diverses sur la production, les principes de fonctionnement de dispositifs techniques, les habitudes de travail, etc. Le tuteur entreprise intervient de temps en temps pour donner quelques conseils et valider les choix. L'apprenti acquiert une grande confiance en lui, ce qui perturbe parfois la relation avec son tuteur, ce dernier considérant qu'il a parfois tendance à aller trop vite. 
TE2 : "Des fois j'ai un peu de mal [...] je sais pas encore quelle est la bonne méthode [...] j'essaie d'y aller progressivement [...] puis au bout d'un moment quand il se braque j'essaie de parler un peu plus directif et en général j'arrête là et puis je reviens un jour ou deux après il a changé d'optique [...] Je pense que $J$ a une forte personnalité et qu'il cherche un petit peu à s'imposer parce qu'il se sent bien » (entretien Tuteur entreprise 2).

Quoi qu'il en soit, à la fin de la formation, l'apprenti est reconnu par ses interlocuteurs comme un ingénieur capable de mener des projets industriels complexes, dans leurs différentes dimensions, en particulier du point de vue du travail relationnel et managérial avec les équipes de production. Il acquiert donc un profil de connaissances tout à fait cohérent avec celui attendu par l'école.

Par contre, il continue à peu jouer le jeu des systèmes didactiques prescrits, en l'occurrence ceux qui sont censés l'amener à davantage de réflexivité (type 5 et 6). Certes, il rend les fiches d'analyse de compétences demandées mais souvent de manière bâclée, ce qui a le don d'énerver son tuteur école. Son mémoire est aussi jugé moyen par le jury final, qui lui reproche un manque de recul et un caractère trop descriptif.

\section{Conclusion : des études de cas paradoxales}

Ces deux études de cas mettent en évidence des réceptions très contrastées des systèmes didactiques prescrits par l'école. Dans le premier cas, on assiste à une bonne mise en œuvre des prescriptions mais la position de l'apprenti à la fin de la formation n'est pas conforme à celle attendue (un ingénieur spécialiste d'un domaine technique). Dans le second cas, la réception des systèmes didactiques prescrits est très mauvaise, mais l'apprenti termine dans une position cohérente avec celle prescrite par l'institution scolaire. Comment expliquer ce paradoxe?

Notre étude montre comment les logiques institutionnelles pèsent sur les tuteurs et les apprentis. Dans le cas 1, la stratégie du tuteur entreprise consiste à utiliser l'affiliation successive de l'apprenti à deux types d'institutions (les ateliers puis le service $R \& D$ ) pour lui faire acquérir les connaissances nécessaires une position de manager de projet, externe à ces institutions. Cette stratégie semble intéressante à la fois parce qu'elle ne lui prend pas beaucoup de temps et parce qu'elle contribue à enrichir l'apprenti de visions différentes de l'entreprise. Mais elle s'avère en réalité problématique. En effet, dans les systèmes didactiques qui se mettent en place au sein de ces deux institutions, les ouvriers, puis le technicien R\&D sont, pendant une longue période (le tuteur entreprise intervient peu) dans une position enseignante dominante vis-à-vis de l'apprenti. Ils ont, de ce fait, tout le temps de lui transmettre des connaissances et des conceptions sur l'entreprise liées à leur position et leur personne. Beaucoup plus tard, quand le tuteur entreprise veut enfin reprendre la main, en initiant lui-même d'autres types de systèmes didactiques visant l'apprentissage du management d'actions d'amélioration, il se heurte à des rapports à des objets peu conformes à ceux attendus d'un manager de projet (par exemple, son rapport de respect de l'autorité des ouvriers), que l'apprenti peine beaucoup à modifier. Pour éviter cette difficulté, une solution aurait été de sortir l'apprenti plus rapidement du service R\&D pour le positionner au sein d'une autre institution, par exemple le service méthodes, plus apte à transmettre des connaissances (notamment sur les objets de l'atelier) et des comportements, conformes avec la position de manager de projets. Cela lui aurait aussi donné une position sans doute plus légitime pour porter des projets de modifications des installations. On aurait pu aussi définir un projet 
portant sur un atelier où l'apprenti n'a pas effectué sa période d'immersion, le libérant ainsi du poids de rapports d'autorité créés avec les ouvriers.

Dans le cas 2, l'apprenti se trouve dès le départ dans une institution type bureau des méthodes, dont la finalité est justement de gérer des projets. Il n'en bouge plus par la suite. Logiquement, il n'a pas les problèmes de son camarade. Mais pourquoi le tuteur est-il si réticent vis-à-vis des prescriptions de l'école? Au-delà d'une simple mauvaise volonté et d'une charge importante de travail dans le service, il y a le fait que l'intégration au sein de cette institution a été pensée et bien organisée par ses responsables. De plus les échanges et les communications dans l'entreprise sont facilités par des environnements de travail peu étendus, une population en moyenne assez jeune et fortement diplômée et l'absence de relations sociales conflictuelles. Tous ces éléments favorisent l'émergence de systèmes didactiques effectifs autour des novices, à leur initiative ou des personnes qui les encadrent. Il n'est donc pas très étonnant que les systèmes prescrits par l'école apparaissent superflus voire nuisibles car concurrents des systèmes effectifs dans l'entreprise. Le deuxième tuteur entreprise est plus ouvert : il comprend mieux l'importance pour l'apprenti d'une cohérence entre son activité en entreprise et le cadre proposé par l'école, notamment pour lui donner une formation plus large sur la conduite de projets industriels. La négociation entre lui et le tuteur école est intéressante car elle aboutit un compromis qui met en valeur ce que peut être un travail d'adaptation des systèmes didactiques prescrits par l'école pour les rendre cohérents et bien articulés avec les logiques de transmission propres aux institutions de l'entreprise.

Au final, cette étude montre que la collaboration entre institution scolaire et professionnelle ne va pas de soi en matière d'organisation pédagogique du parcours en entreprise. Le jeu en vaut-il la chandelle? Oui car ces études de cas montrent que l'école peut avoir une action didactique décisive : orienter vers des objets peu visibles à partir d'une position d'acteur opérationnel, comme par exemple le processus de production ; construire un projet peut-être moins canonique du point de vue des ingénieurs, mais plus favorable à l'apprentissage ; favoriser l'acquisition de méthodes de retour réflexif sur l'action; etc. Par contre, il apparait clairement contre-productif d'appliquer une organisation prescrite du parcours en entreprise de manière rigide. Pour chaque nouvelle entreprise, un travail est nécessaire pour analyser les logiques de transmission propres aux institutions qui accueillent l'apprenti, puis pour concevoir des parcours cohérents et efficaces. Les institutions scolaires ont donc moins intérêt à bâtir un cadre prescriptif rigide, qu'un ensemble de dispositifs et de ressources didactiques adaptables selon les cas. La limite est qu'une telle perspective demande une expertise importante, que la plupart des tuteurs école et entreprise n'ont pas. On peut cependant concevoir des formations pour les sensibiliser à ces questions, comme nous l'expérimentons depuis quelques années dans notre institution de formation de techniciens supérieurs.

Sur le plan scientifique, notre approche diffère d'autres courants de recherche, tels la didactique professionnelle (Pastré, Mayen, Vergnaud, 2006) ou le « workplace learning » (Lave \& Wenger, 1991 ; Darrah, 1996; Billett, 2006), sur le plan de l'objet d'étude. Notre intérêt va vers la transmission de connaissances organisée intentionnellement en situation de travail et à la collaboration entre institutions scolaires et professionnelles pour organiser ce type de transmission, alors que les courants de recherche cités ci-dessus ne s'intéressent qu'aux apprentissages dans le travail ou dans la participation à des communautés de pratiques. Par ailleurs, notre étude se déploie sur un temps long pour pouvoir analyser des évolutions 
significatives vers un type de métier, ce qui nous différencie à nouveau de la didactique professionnelle, privilégiant des analyses fines de l'activité, mais nous rapproche des études sur « workplace learning » ou la théorie de l'activité (Tuomi-Grohn \& Engenström, 2003) qui elles aussi privilégient des études longues pour les mêmes raisons.

L'intérêt spécifique du cadre théorique proposé est de conduire à une mise en valeur les logiques de transmission institutionnelles sur un lieu de travail, et de comprendre comment celles-ci contraignent tant ceux qui jouent un rôle de formateur, que ceux qui sont en position d'apprenant. A contrario, la théorie institutionnelle apparait sans doute moins convaincante pour saisir des facteurs plus individuels dans les processus de transmission et d'apprentissage. Cette remarque conduit probablement à nuancer les interprétations proposées plus haut par les différences de personnalité et d'expériences des tuteurs et des apprentis. En ce qui concerne les apprentis, leurs expériences formatives antérieures les conduisent sans doute à tirer un profit différent des différents systèmes didactiques effectifs. Le premier apprenti est par exemple très attiré par la culture technique papetière. Il a reçu une formation très technique dans ce domaine, ponctuée par un stage d'étude sur les eaux de rejets d'une papeterie très éloigné du management. A l'inverse, le second apprenti ne cache pas son ras le bol des bancs de l'école et des approches trop théoriques et à l'inverse, son goût pour l'action et la communication sur le terrain. Sa formation en génie mécanique, complétée par un stage en bureau d'étude, lui donne aussi des atouts importants pour tirer partie des connaissances transmises par les acteurs de l'entreprise. En ce qui concerne les tuteurs entreprise, le fait qu'ils soient très différemment convaincus par les dispositifs créés par l'école a peut-être à voir avec leur propre expérience de formation initiale ou continue. Par exemple, dans le cas 1, le tuteur explique que l'absence de stage ouvrier lui a été très préjudiciable dans sa propre formation d'ingénieur. Dans le cas 2 , le second tuteur entreprise est devenu ingénieur après une formation continue en alternance et garde le souvenir d'une négociation importante de ses missions entre son entreprise et le centre de formation. Il faudrait donc disposer d'une étude plus conséquente sur les biographies des apprentis et des tuteurs pour pouvoir mieux comprendre les effets de ces histoires singulières sur leur participation aux processus de transmission et d'apprentissage.

\section{Bibliographie}

Billett, S. (2000). Guided learning at work. Journal of Workplace Learning, 12(7), 272-285.

Billett, S. (2006). Constituting the workplace curriculum. Journal of curriculum studies, 38(1), $31-48$

Chaix, M.-L. (2000). Se former en alternance. Le cas de l'enseignement technique agricole. Paris: L'Harmattan.

Chevallard, Y. (1992). Concepts fondamentaux de la didactique : perspectives apportées par une approche anthropologique du didactique. Recherche en didactique des mathématiques, Vol. 12(1), 73-112.

Chevallard, Y. (2003). Approche anthropologique du rapport au savoir et didactique des mathématiques. In S. Maury \& M. Caillot (Eds.), Rapport au savoir et didactiques (pp. 81-104). Paris.

Cohen-Scali, V. (2000). Alternance et identité professionnelle. Paris: PUF.

Besson, M., Collin, B., Geay, A., \& Hahn, C. (2005). Pour une nouvelle approche de l'alternance dans l'enseignement supérieur. In C. Hahn, M. Besson, C. Béatrice \& A. Geay (Eds.), 
L'alternance dans l'enseignement supérieur. Enjeux et perspectives (pp. 15-29). Paris: L'Harmattan.

Darrah, C. N. (1996). Learning at work: an exploration in industrial ethnography. New York: Garland.

Douglas, M. (1987). How institutions think? Londres: Routledge \& Kegan Paul.

Durkheim, E. (1998). De la division du travail social : étude sur l'organisation des sociétés supérieures (1 ère édition : 1893, Alcan éditeur ed.). Paris: PUF.

Geay, A. (2000). L'école de l'alternance. Paris: L'harmattan.

Lave, J., \& Wenger, E. (1991). Situated Learning : legitimate peripheral participation. Cambridge: Cambridge University Press.

Leplat, J., \& Hoc, J.-M. (1983). Tâche et activité dans l'analyse psychologique des situations. In J. Leplat (Ed.), L 'analyse du travail en psychologie ergonomique. (Vol. 1). Toulouse: Octares.

Lerbet, G. (1993). Alternance et cognition. Education permanente, 115, 65-77

Maubant, P. (1994). Métacognition, transfert de compétence et pédagogie de l'alternance. Université de Rouen, Rouen.

Pastré, P., Mayen, P., \& Vergnaud, G. (2006). La didactique professionnelle. Revue Française de Pédagogie, 154, 145-198.

Pelpel, P., \& Troger, V. (1993). Histoire de l'enseignement technique. Paris: Hachette.

Theureau, J. (1991). Les raisonnements dans le travail. In R. Amalberti, M. De Montmollin \& J. Theureau (Eds.), Modèles en analyse du travail. Liège: Pierre Margada.

Tiberghien, A. (1997). Learning and teaching: differentation and relation. Research in Science Education, 27(3), 359-382.

Tuomi-Grohn, T., \& Engenström, Y. (2003). Between school and work: new perspectives on transfer and boundary-crossing. Amsterdam: Pergamon.

Veillard, L. (2000). Rôle des situations professionnelles dans la formation par alternance. Cas des élèves-ingénieurs de l'ISTP de Saint-Etienne", Mémoire de Thèse, Université Lumière Lyon 2, Lyon.

Veillard, L. (2004). Le tutorat à l'épreuve des spécificités sociales et techniques de l'entreprise. Education Permanente, 159,117-138. 